\title{
AR Based App for Tourist Attraction in ESKİ ÇARŞI (Safranbolu)
}

\author{
Merve POLAT ${ }^{1}$, Ismail Raklp KARAŞ ${ }^{2}$, İdris KAHRAMAN ${ }^{3}$, Behnam ALIZADEHASHRAFI ${ }^{4}$ \\ 1,2,3 Karabük University, Computer Engineering Department, Karabük, \\ 1․mmervepolat@ hotmail.com, ${ }^{2}$ ismail.karas@ karabuk.edu.tr, ${ }^{3}$ idriskahraman@karabuk.edu.tr \\ ${ }^{4}$ Tabriz Islamic Art University,Tabriz, Iran, ${ }^{4} \underline{b}$.alizadehashrafi@ tabriziau.edu.ir
}

\begin{abstract}
This research is dealing with 3D modeling of historical and heritage landmarks of Safranbolu that are registered by UNESCO. This is an Augmented Reality (AR) based project in order to trigger virtual three-dimensional (3D) models, cultural music, historical photos, artistic features and animated text information. The aim is to propose a GIS-based approach with these features and add to the system as attribute data in a relational database. The database will be available in an AR-based application to provide information for the tourists.
\end{abstract}

Keywords: Safranbolu, ESKİ ÇARŞI, Augmented Reality, 3D modelling, 3D GIS

\section{INTRODUCTION}

In fact Augmented Reality (AR) is a live view of real-world environment with some augmented elements such as music, movie, graphics, 3D models, GPS data and etc. AR can be used for tourist attraction as a main issue of economy especially in Turkey. It can be utilized to increase the level of interaction, detection and guidance. It is possible to implement AR with printed documents along with some markers, targets, or specific characters as a triger for starting the process of augmenting on Android/ IOS based gadgets. In the other word AR is a process of overlapping the real world with computer-generated elements. It provides the position for virtual materials created in computer environment like three dimensional (3D) structures, animations, and various virtual materials in the real worldThese elementsenrich the real world and make it more useful and functional and also attractive for the tourists.

Currently the AR-based applications are widely used in different fields such as engineering, architectue, advertising, games, education and medical tutorials for the students and it will be increased tremendosly in the near future.

Developing applications for mobile devices have been growing rapidly since 2000 exactly after the invention of the smart phones. The usage of GPS started in 1993, passing from barcode to quick response $(\mathrm{QR})$ code by Jun Rekinimo in 1996 and then the first GSM phone with the integrated GPS came out. In 1999 the wireless network (Wi-Fi) protocol was introduced by Benefon that caused the first commercial cell phone camera by Sharp in 2000 . Since that time the usage of augmented reality applications was rocketed amazingly. One of the first examples is BatPortal which was developed in 2001 as a wireless Personal Digital Assistant (PDA) based on augmented reality system. The process of detecting the location was done by ultrasonic monitoring system called "Bats". The system was utilized in two ways as a head-mounted viewer along with a portable computer and a display augmented world using PDA. The cause of gaining an importance in the historical development of BatPortal was the usage of PDA in this system operatively(Hill, 2013).

Mathias Möhring implemented the first video-based augmented reality system for mobile devices in 2004. The system was running through 3D markers via operated live video stream. (Mistry, 2009) (Hill, 2013).
In 2009, Pranav Mistry implemented an AR project as a "Sixth Sense" in MIT Media Laboratory. Mistry developed a wearable tool consisting of mirror, camera, mobile device, colored markers and projection which can use any surface like wall, paper and even palms of hand as an interface. All hand movements and gestures (enlargement, reduction, and drag-and-drop) on the touch screen could be done on any surface. Hand movements and gestures could be recognized via fixed camera hanging on the neck and some algorithms coded by Mistry. Mistry in his TED talk in 2009 has indicated that this project will help us to be more connected to our physical world by adding the sixth sense to our five senses and to stay like human rather than a machine. He claimed that his software would be available as an open source application for sharing (Mohring et al., 2004).

The first instance of smart glasses that Google has worked for many years was introduced in 2012. Google Glass is allowing users to control the device with voice and touchpad located on the side of Google Glass. Glass can be used with voice command for capturing photos, recording video, video calling via Hangout Application via wireless network, send audio questions and response instantly from Google source engine. After connecting to a smartphone via Bluetooth, it can utilize the wireless network and GPS, it can make a call, it can send a text message and it can use all of the functions that are available on the mobile phone. Google Glass has become a controversial issue because of very small screens and harmful radiations emitted from glasses and has brought some problems in terms of ethics, privacy and security. At this point, due to losing interest, users have abandoned it units the Google X (The "Explorers" version) was stopped sales in January 19, 2015 and it was planned to be redeveloped with more advanced features(Newman et al., 2001) . A lot of smart glasses have been put on the market by the some companies and inventors before and after Google Glass. Vuzix, Recon, Oculus, Meta and many firms besides Google are struggling to develop enhanced and AR glasses (Clemens Arth and Alessandro Mulloni, 2015).

AR technology is currently used in a number fields, such as medicine, education and simulated training among others (Yu et al., 2009). It is also used within the tourism sector, aiming to improve the tourist experience. Besides, several examples have shown that AR can assist tourist 
organizations and professionals towards reaching a wider audience by serving as the delivery technology of appealing multimedia content and mobile applications. On the other hand, AR information systems can help tourists in accessing semantic information and improving their knowledge regarding a touristic attraction or a destination, while enhancing the tourist experience and offering increased levels of entertainment throughout the process (Fritz et al., 2005). Most importantly, such information systems are able to personalize the delivery of the multimedia content according to the user's characteristics and the use context, thus supporting their deployment for a number of scenarios.

In terms of "Augmented Reality Application for Safranbolu Eski Çarşı " Project, it is aimed to give ESKİ ÇARȘI map for those who want to get detailed information about the structure of Safranbolu and intended to provide necessary information. This project provides useful information for domestic and foreign tourists, researchers, and people who cannot visit physically can have easy access to historical, spatial definition of cultural fields, images in all angles, 3D models, videos, and cultural details about Safranbolu. In this project 3D building structures and the information received from different historical points will be brought together in augmented reality project and it will give historical and semantic information by developing mobile application. In the next part, the focus will be on workflow and the steps that will be completed.

\section{MATERIALS AND METHODOLOGY}

Actually in AR-based project the idea is scanning the images, processing the images and finally matching the result with image marker or target within the database and in case of equality, the application will trigger for popping up some information.

The application will become active according to the specified marker or GPS location. Thus, the user can reach the whole data about the application within the database via user-friend interface.

The steps towards the realization and implementation of GIS-based AR for Safranbolu Eski Çarşı historical point of interests are as follows:

- $\quad$ Taking snapshots from building facade.

- Editing the images with some additional photo editor software.

- Modeling 3D structures and texturing 3D model facades with edited images.

- Importing 3D models into Unity3D environment and develop the AR application via Unity and Voforia.

- Obtaining semantic information about the 3D models and combine them in the spatial database.

- Developing android-based AR application for viewing generated $3 \mathrm{D}$ models.

\subsection{Taking Photos of Building Facades}

The texturing process can be performed after designing 3D solid models to increase the Level of Realisim(LoR)(Alizadehashrafi, 2015). Texturing process has been done with digital images, plain color, and shaded colors. In order to take facade images, high-resolution digital camera Canon EOS 7D with 18MP APS-C CMOS sensor was used (see Figure 1.). Snapshots for facades should be done with a perpendicular angle to reduce leaning problems of deeper or outer geometreis. Taking photos for all facades of buildings from different angles can be used for $3 \mathrm{D}$ solid modeling and provide details of the structure precisely. In order to have a small data size on the hard disk drive, images should be created in jpg and png (suports Alpha Chanel and transparency) formats.

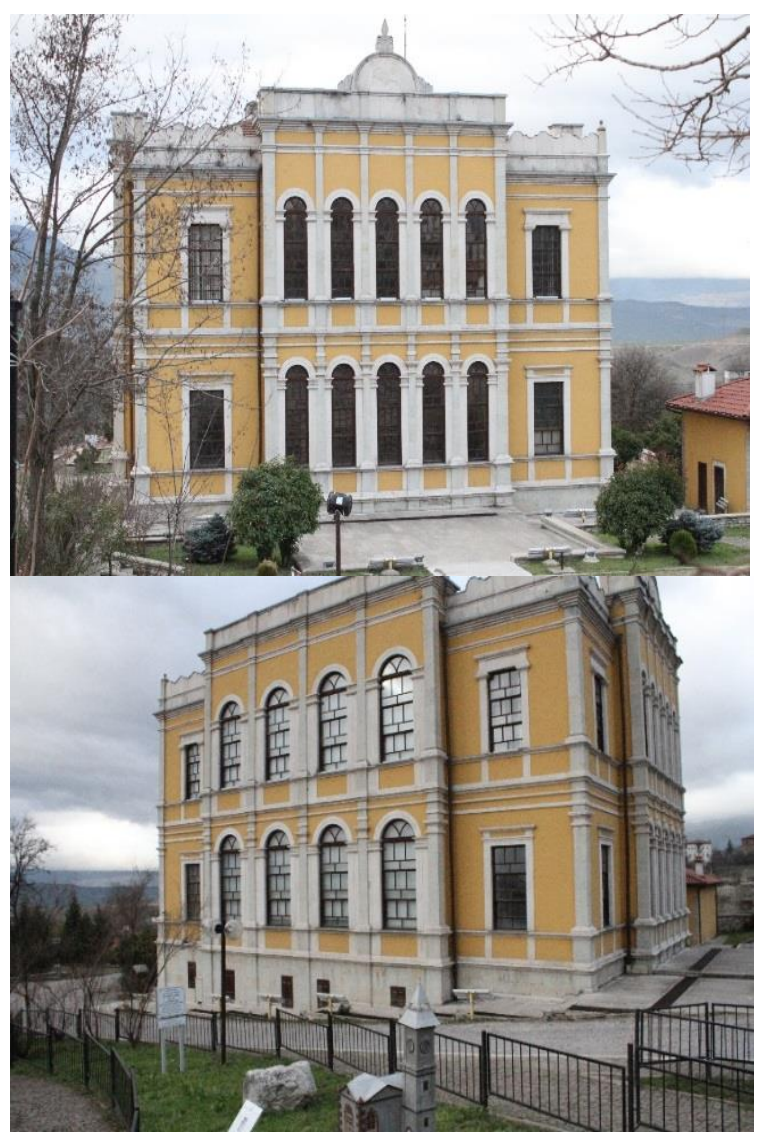

Figure 1. Historical Musium of Safranblue (test bed)

\section{2. Editing the Captured Photos with Additional Software}

Some objects like cars, pedestrians, shadows and any other obstacles in front of the facade can cause lack of quality on the final 3D model (Alizadehashrafi et al., 2009). Unwanted objects may cause visual pollution and realistic view of the 3D model cannot be achieved. Therefore, cleaning unnecessary parts on the obtained images using Photoshop editor should be made in advance via different tools such as free transform, lef2right and up2down transformation and etc. (Alizadehashrafi and Rahman, 
2013) In some cases perpendicular photos from the facades cannot be taken due to the nature of structures and obstacles and some adjustments should be made on them. Photoshop Perspective Crop Tool is used for parallel transformation over these images (See Figure 2)..

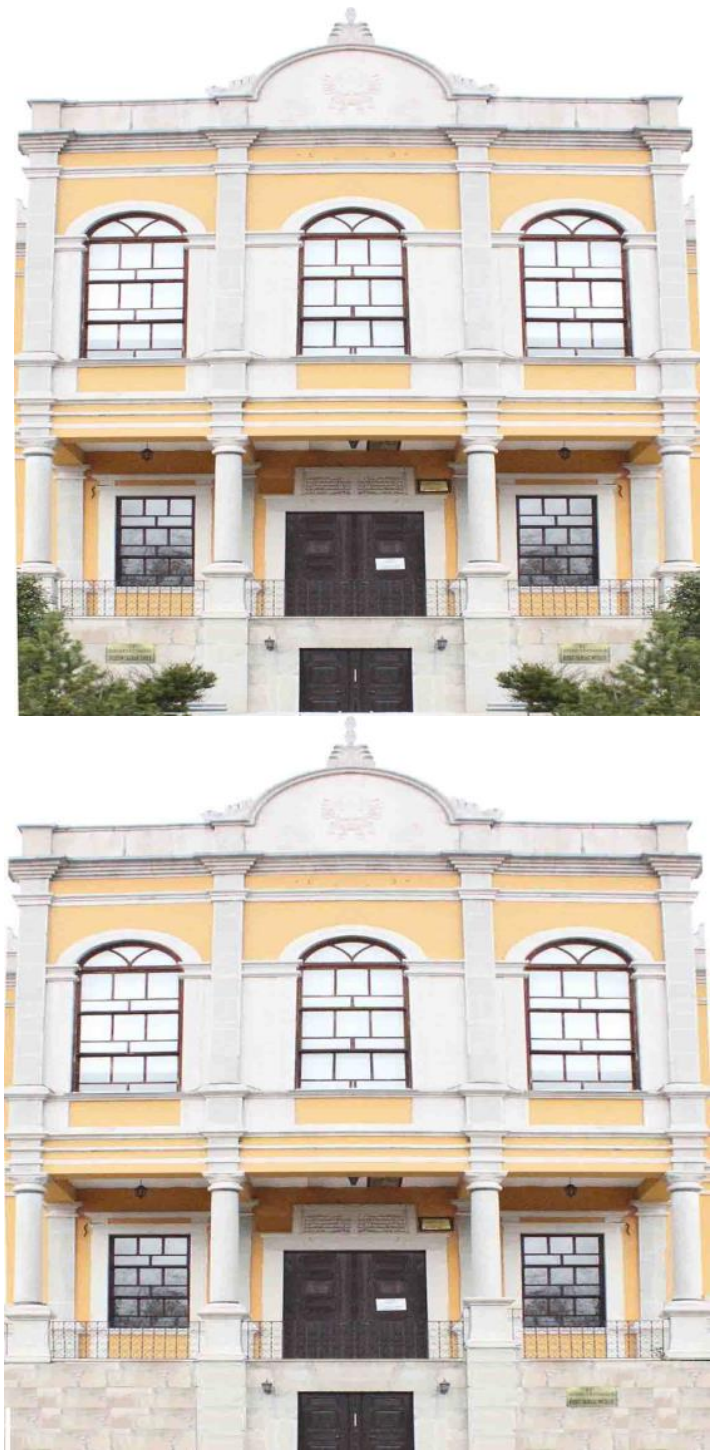

Figure 2. Cleaning the Image with Photoshop Editor

\subsection{B Modellerin Üretilmesi ve Düzenlenen Fotoğrafların 3B Modellere Giydirilmesi}

The most important POIs in the Safranbolu Eski Çarşı were modeled precisely in LoD3 and textured properly to increase LoR with very small data size using SketchUp Software (See Figure 3).
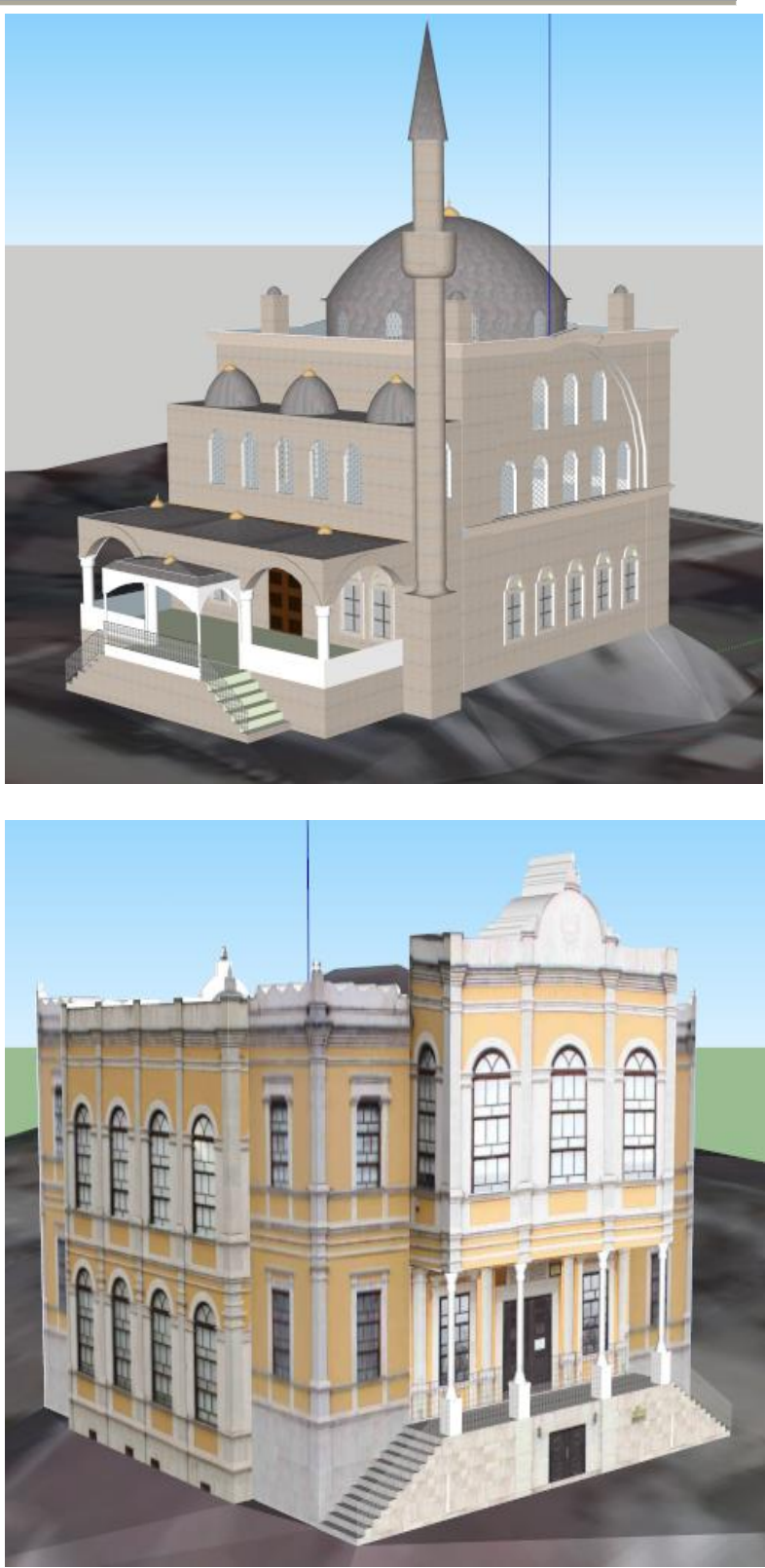

Figure 3. 3D Architectural Models

\subsection{Importing 3D Models into Unity3D and} Implementation of AR app with Unity

By utilizing Unity3D game engine it can ease and improve the AR project and it is possible to export apps for different mobile operating systems such as IOS and android. Programming languages for developing games have various libraries and lots of features can be applicable easily. Another facility provided by the Unity3D to game developers is to compile the game without any changes in the game infrastructure on different platforms such as PC, Mac, Web, iOS, Android and Windows Phone. One of the significant advantages of the Unity3D game engine comparing to other game engines is making the ability for the developers to write program code during the game development process in different languages such as C\#. The majority of the game engines separate graphics and code section but in Unity3D both run together. This logic 
provides flexibility to developers to shorten the development time.

Unity3D game engine supports 3D models in FBX or OBJ formats. The 3D models created in different software can be imported to Unity3D. In this project 3D models that were generated in SketchUp can be loaded on this platform (See Figure 4). In order to avoid losing textures and geometries and also some distortions like exterior coloring, it is recommended to import the 3D models into Unity3D in FBX format.

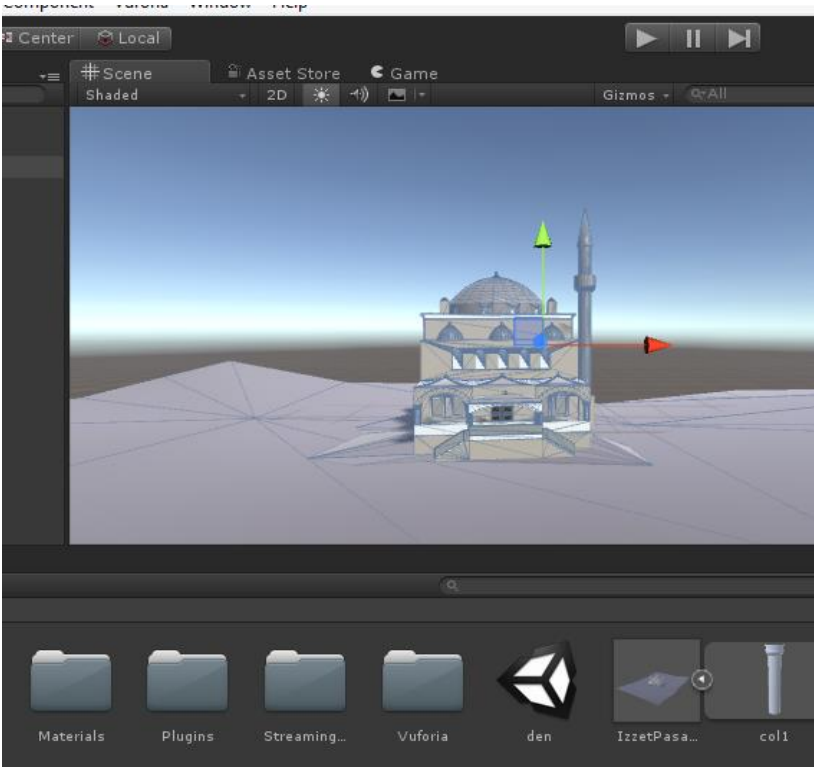

Figure 4. Importing 3D Models into the Unity Environment

\subsection{Obtaining Semantic Information of 3D Models and Combining in a Spatial Database}

Some information such as 3D views, historical information, videos, audio files and location information and etc. that are related to the 3D models were created or collected from different resources. The created geospatial database about the 3D models can be brought together in an AR-based user-friend android or IOS application.

\subsection{Developing Android-based Mobile Application for Running 3D Models}

In this research project Android Technology is used to develop AR-based app for the users and tourists. The users will have easy access to the information about the specific POIs with Android-based user-friend interface. The mobile devices of the users can receive the necessary information and app via $\mathrm{WiFi}$ or Bluetooth system and present adequate information with the recognition of the POIs and extract the data from the relevant database. Users can receive lots of information about the 3D models, historical photographs, cultural music, and artistic animation text easily.

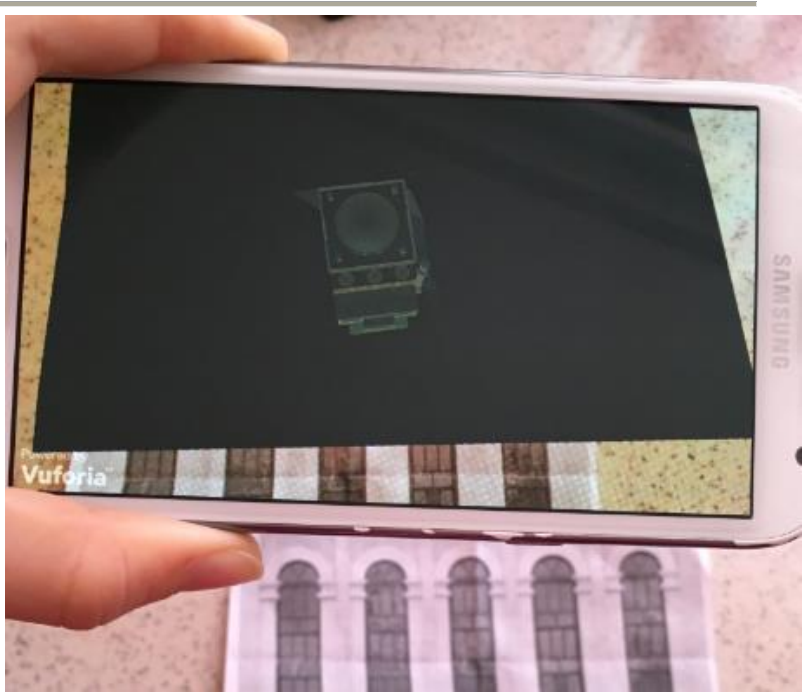

Figure 5. Mobile Application Development

\section{RESULTS}

The main objective of this paper was to provide the current implementation of AR in tourism context and identify areas of research and development that is required to guide the early stages of AR implementation in a purposeful way to enhance the tourist experience and benefit tourism business.

The protection of historical and heritage landmarks and passing the concept to the new generations in the future, are vital for Safranbolu as these POIs aredetermined and registerd by UNESCO. In fact Safranbolu is a world city with three thousand years of history and nature as well as wooden three floor structures, culture, ethnographic structure, stone and artistic features. An android-based augmented reality project is aimed in accordance with Safranbolu. This project provides lots of information for domestic and foreign tourists, researchers, and those people who cannot visit Safranbolu phisycally.

Lastly but importanly, some features should be existed in the AR applications like providing access to location-based information, related to the immediate surroundings of tourists, enabling access to multivariable content, being operable in terms of representing text, video, or images

In the future work of this research to increase the effience of the AR based application. One of them is to reach semantic and detailed information about specific parts of the structures. Also a feature by choosing special marked positions in the area to virtually zoom there and offer a panoramic view from that position, in which the user can navigate using the binoculars can be added.

\section{ACKNOWLEDGEMENT}

This study was supported by Karabük University, Department of Scientific Research Projects (Project No: KBÜ-BAP-16/1-YL-140) research grant. We are indebted for its financial support. 


\section{REFERENCES}

ALIZADEHASHRAFI, B. 2015. Using Parameters of Dynamic Pulse Function for 3d Modeling in LOD3 Based on Random Textures. ISPRSInternational Archives of the Photogrammetry, Remote Sensing and Spatial Information Sciences, 51-55.

ALIZADEHASHRAFI, B. \& RAHMAN, A. A. 2013. Towards Enhancing Geometry Textures of 3D City Elements. Developments in Multidimensional Spatial Data Models. Springer.

ALIZADEHASHRAFI, B., RAHMAN, A. A., COORS, V. \& SCHULZ, T. 2009. 3D navigation systems based on synthetic texturing.

CLEMENS ARTH, R. G., LUKAS GRUBER, TOBIAS LANGLOTZ, \& ALESSANDRO MULLONI, D. S., DANIEL WAGNER 2015. The History of Mobile Augmented

Reality.

FRITZ, F., SUSPERREGUI, A. \& LINAZA, M. T. Enhancing cultural tourism experiences with augmented reality technologies. 2005. 6th International Symposium on Virtual Reality, Archaeology and Cultural Heritage (VAST).

HILL, S. 2013. A look at all the Google Glass competitors in development. Retrieved April, 28, 2014.

MISTRY, P. 2009. The thrilling potential of SixthSense technology.

MOHRING, M., LESSIG, C. \& BIMBER, O. Video seethrough ar on consumer cell-phones. Proceedings of the 3rd IEEE/ACM International Symposium on Mixed and Augmented Reality, 2004. IEEE Computer Society, 252-253.

NEWMAN, J., INGRAM, D. \& HOPPER, A. Augmented reality in a wide area sentient environment. Augmented Reality, 2001. Proceedings. IEEE and ACM International Symposium on, 2001. IEEE, 77-86.

YU, D., JIN, J. S., LUO, S., LAI, W. \& HUANG, Q. 2009. A useful visualization technique: a literature review for augmented reality and its application, limitation \& future direction. Visual information communication. Springer. 